\title{
Efecto de año, bimestre y número de parto de la cerda en el tamaño y peso de la camada al nacer y al destete en una granja comercial.
} Artículo Original

\author{
Mario Gómez Medina ${ }^{1}$ José C. Segura-Correa² ${ }^{\text {, Jorge C. Rodríguez-Buenfil² }}$.
}

${ }^{1}$ Univasa S.A. de C.V. Avenida Principal x José María Castro Tejero. Cd. Industrial, ${ }^{2}$ Facultad de Medicina Veterinaria y Zootecnia, Universidad Autónoma de Yucatán, Apdo. postal 4-116 Mérida, Yucatán, México.

\section{RESUMEN.}

Introducción. Investigaciones realizadas en varios países, han demostrado que la producción de la hembra está influenciada por el año o época de parto y por el número de parto de las marranas. Este efecto se manifiesta en los distintos indicadores de producción, como fertilidad, tamaño y peso de la camada al nacer y al destete. Conocer la magnitud del efecto del número de parto en los rasgos predestete, es de gran importancia para tomar decisiones sobre el sistema de desecho y reemplazo de la piara, lo cual conduciría a la toma de decisiones más adecuadas al sistema de manejo en la granja de interés. El objetivo de este estudio fue determinar los efectos de año, bimestre y número de parto de la marrana en algunos indicadores predestete de una granja porcina del estado de Yucatán, México.

Material y métodos. Se analizaron los registros de dos años de producción de una granja localizada en la zona centro del estado de Yucatán, con un clima tropical subhúmedo. Datos de 8570 y 7841 tamaños de camada y promedios de peso de los lechones al nacimiento y al destete, respectivamente, fueron utilizados. La información mensual de las marranas se combinó para formar seis bimestres. El tamaño de camada al nacer se definió como el número total de lechones al nacer (vivos y muertos). El modelo que describió a las variables de respuesta incluyó los efectos fijos de año de parto, bimestre de parto y número de parto de la marrana.

Resultados. Las medias generales y desviaciones estándares para el tamaño de la camada al nacer (TCN), número de lechones nacidos vivos (NLV), promedio de peso de los lechones al nacer (PLN), número de lechones destetados (NLD) y peso promedio de los lechones al destete (PLD) fueron de $10.99 \pm 2.93$ lechones, $10.24 \pm 2.83$ lechones, $1.58 \pm 0.25 \mathrm{~kg}, 9.44 \pm 1.11$ lechones y $5.81 \pm 0.89 \mathrm{~kg}$, respectivamente. Se encontraron efectos $(\mathrm{p}<0.05)$ de año, bimestre y número de parto en el TCN y NLV, pero no del año de parto en PLN. Con respecto al NLD se encontró efecto de año y bimestre

Solicitud de sobretiros: M. en C. Jorge C. Rodríguez-Buenfil, Depto. de Epidemiología, Facultad de Medicina Veterinaria y Zootecnia, Universidad Autónoma de Yucatán, Apdo. Postal 4-116, C.P. 97100, Mérida, Yucatán, México. Recibido el 12/Junio/1998. Aceptado para publicación el 1/Sep./1998. E-mail: rbuenfil@tunku.uady.mx 
M Gómez Medina, JC Segura-Correa, JC Rodríguez-Buenfil.

de parto $(\mathrm{p}<0.05)$, pero no del número de parto ( $>0.05)$; mientras que el PLD se vió influenciado por los tres factores estudiados $(\mathrm{p}<0.05)$. En general los indicadores productivos para el año de 1995 fueron mejores que para 1994. El efecto de bimestre fue significativo $(\mathrm{p}<0.05)$ sobre todas las variables de respuesta estudiadas, sin embargo, no se observó ninguna tendencia. Las marranas de primer y segundo parto tuvieron camadas con pesos menores que aquellas con 3 o 4 partos. Sin embargo, al destete no se encontró diferencia ( $>0.05$ ) en las medias de los tamaños de camada $\mathrm{y}$ con respecto a los PLD las marranas de primer y cuarto parto tuvieron los promedios más bajos $(\mathrm{p}<0.05)$.

Discusión. Los PLN y PLD estuvieron influenciados por el número de parto de las marranas; mientras que el año y bimestre de nacimiento fueron importantes fuentes de variación para todas las variables de respuesta estudiadas.

(Rev Biomed 1999; 10:23-28)

Palabras clave: Porcicultura, camada.

\section{SUMMARY.}

Year, bi-monthly and parity effects on litter size and weight at birth and at weaning on a commercial farm.

Introduction. Research in some countries has demonstrated that sow production is affected by year or season of farrowing and by the sow's parity number. The effects are manifested in the different indicators of production, such as: fertility, and size and weight of the litter at birth or at weaning. It is important to know magnitude of the effect of parity number on preweaning traits in order to make decisions on the culling and replacement systems, therefore enabling appropiate decisions on the management system in the farm to be taken. The objective of this study was to determine the effect of parity number of the sow, and yearly and bimonthly effects on preweaning traits on a commercial farm in Yucatan, Mexico.
Material y methods. Two annual records from a farm located in the central region of the state of Yucatan, Mexico were used. 8570 y 7841 data on litter size and weight of the piglets at birth and at weaning, respectively, were analysed. Monthly records were combined to obtain six bi-monthly periods. Litter size at birth was defined as the total number of piglets born (dead and alive). The statystical model which described the response variables included the effects of year, bi-monthly and sow's parity number.

Results. The general means and standard deviations for litter size at birth (LSB), number of piglets born alive (NBA), piglets average weight at birth (AWB), number of piglets weaned (NPW) and piglets average weight at weaning (AWW) were: $10.99 \pm 2.93$ piglets, $10.24 \pm 2.83$ piglets, $1.58 \pm 0.25 \mathrm{~kg}, 9.44 \pm 1.11$ piglets y $5.81 \pm 0.89 \mathrm{~kg}$, respectively. Sow's parity, number, year of farrowing and bi-month of farrowing effects were found on LSB and NBA, however year of farrowing had no effect on AWB. With respect to NPW only yearly and bi-monthly effects were found $(p<0.05)$. AWW was affected by the three factors studied $(\mathrm{p}<0.05)$. In general, the means for the preweaning traits were better in 1995 than in 1994. Bi-monthly effect did not show any trend over time. Fist and second farrowing sows had lighter litters than those with 3 or 4 farrowings. However, at weaning, sows had similar litter sizes. Sows with 1 or 4 farrowing had the lowest AWW ( $\mathrm{p}<0.05)$. Discusion. AWB and AWW were influenced by the sow's parity number, whereas year and bimonth were important sources of variation for all the variables studied. (Rev Biomed 1999; 10:23-28)

Key words: Swine production, litter.

\section{INTRODUCCIÓN.}

Investigaciones realizadas en varios países, han demostrado que la producción de la marrana está influenciada por el número de parto. Este efecto se manifiesta en los distintos componentes de

\section{Revista Biomédica}


Factores asociados a la producción de cerdos.

producción, como fertilidad, tamaño y peso de la camada al nacer y al destete (1-3). Dicho comportamiento productivo, es según dichos autores, debido a que la hembra alcanza su mayor producción al llegar a la madurez física, lo cual sucede al cuarto o quinto parto, después de los cuales empieza a descender debido a la edad del animal.

Estudios realizados en México confirman este comportamiento en las marranas (4-10). Conocer la magnitud del efecto que tiene el número de parto sobre las características predestete, es de gran importancia para tomar decisiones sobre el sistema de desecho y reemplazo de la piara, lo cual conduciría a la toma de decisiones más adecuadas al sistema de manejo en la granja de interés (11).

Otro factor importante es el mes o época del año los cuales según algunos autores influyen también sobre la eficiencia reproductiva de las cerdas. Rico (12) en Cuba encontró que la época del año afectó $(\mathrm{p}<0.05)$ el tamaño y peso de la camada al nacer y al destete. En México, Segura y Segura (9) encontraron diferencias atribuibles a la época de servicio. De aquí la importancia de establecer los meses o épocas más críticos del año para tomar los cuidados necesarios, para reducir los efectos de temperatura y humedad normalmente asociados a esos meses.

Por lo anterior, se desarrolló un estudio observacional para obtener indicadores productivos predestete de las marranas de acuerdo al número de parto, año y época de parto en una granja modelo localizada en el estado de Yucatán.

\section{MATERIALES Y MÉTODOS.}

Se utilizaron los registros de producción de las marranas parida en 1994 y 1995 en una granja comercial, localizada en la zona centro del estado de Yucatán. La región tiene un clima tropical subhúmedo con lluvias en verano, con promedio mensual de temperatura de $26^{\circ} \mathrm{C}$.

Las marranas se alimentaron dos veces al día, con una ración comercial a base de soya y sorgo, con un $14 \%$ de proteína, y con libre acceso al agua.
Cada marrana recibió dos montas, una en la mañana y otra en la tarde. Una vez servidas, las marranas permanecían en jaulas, en el área de gestación temprana, hasta realizar el diagnóstico de gestación a la quinta semana. Las marranas gestantes se trasladaron a jaulas en el área de gestación definitiva hasta cinco días antes del parto, fecha en que fueron trasladadas a jaulas de maternidad, donde permanecieron hasta el destete ( $21 \pm 3$ días).

Se utilizaron los datos de 8570 tamaños y promedios de peso de los lechones al nacer y 7841 tamaños y promedios de peso de los lechones al destete, capturados en el programa PigCHAMP (v 3.0 Universidad de Minnesota 1991). Los datos mensuales de las marranas se combinaron para formar seis bimestres. El tamaño de camada al nacer se definió como el número total de lechones al nacer (vivos y muertos).

Para describir las características predestete, se utilizó un modelo estadístico de efectos fijos que incluyó como fuentes de variación al año de parto, bimestre de parto y número de parto de la marrana. Todos los análisis se realizaron con el procedimiento GLM (SAS 1989).

\section{RESULTADOS.}

Las medias generales y desviaciones estandares para el tamaño de la camada al nacer (TCN), número de lechones nacidos vivos (NLV), promedio de peso de los lechones al nacer (PLN), número de lechones destetados (NLD) y peso promedio de los lechones al destete (PLD) fueron de $10.99 \pm 2.93$ lechones, $10.24 \pm 2.83$ lechones, $1.58 \pm 0.25 \mathrm{~kg}, 9.44 \pm 1.11$ lechones y $5.81 \pm 0.89 \mathrm{~kg}$, respectivamente.

Se encontraron efectos significativos ( $p<0.01)$ de año, bimestre y número de parto para el TCN y NLV, pero no del año de parto sobre PLN (cuadro 1). Con respecto al NLD se encontró efecto significativo $(\mathrm{p}<0.01)$ de año y bimestre de parto, pero no del número de parto; mientras que el PLD fue influenciado por los tres factores estudiados (cuadro 1). Las medias de cuadrados 
M Gómez Medina, JC Segura-Correa, JC Rodríguez-Buenfil.

\section{Cuadro 1}

Análisis de varianza para algunos indicadores predestete de lechones en una granja comercial de Yucatán.

\begin{tabular}{|c|c|c|c|c|c|c|}
\hline $\mathrm{FV}$ & GL & $\mathrm{TCN}$ & NLV & PLN & ND & PLD \\
\hline Año de par & rto 1 & $466.59 *$ & $452.56^{*}$ & 29.93 & $64.91 *$ & $534.64 *$ \\
\hline $\begin{array}{l}\text { Bimestre } \\
\text { de parto }\end{array}$ & 5 & $82.46 *$ & $79.72 *$ & $579.81^{*}$ & $37.06^{*}$ & $196.47 *$ \\
\hline $\begin{array}{l}\text { Número } \\
\text { de parto }\end{array}$ & 3 & $322.87 *$ & $156.34 *$ & $11183.46^{*}$ & 0.66 & $22.45^{*}$ \\
\hline Error & $\begin{array}{l}8560 \\
7481\end{array}$ & 8.59 & 8.03 & 61.69 & 1.23 & 0.79 \\
\hline $\mathrm{R}^{2}$ & & 4.56 & 3.44 & 8.60 & 5.20 & 30.38 \\
\hline $\mathrm{CV}$ & & 26.66 & 27.66 & 15.67 & 11.72 & 15.34 \\
\hline
\end{tabular}

$\mathrm{TCN}=$ tamaño de la camada al nacer, $\mathrm{NLV}=$ número de lechones nacidos vivos, $\mathrm{PLN}=$ promedio de peso de los lechones al nacer, NLD= número de lechones destetados, y PLD peso promedio de los lechones la destete.

$* \mathrm{p}<0.01$

\section{Cuadro 2}

Medias de cuadrados mínimos para tamaño de camada y peso de los lechones al nacer y al destete según el año y bimestre de nacimiento.

\begin{tabular}{lrrrrrrl}
\hline & $\mathrm{N}$ & $\mathrm{TCN}$ & $\mathrm{NLV}$ & $\mathrm{PLN}$ & $\mathrm{N}$ & NLD & PLD \\
\hline Año de parto: & & & & & & & \\
$\quad 1994$ & 5544 & $10.96 \mathrm{a}$ & $10.15 \mathrm{a}$ & $1.60 \mathrm{a}$ & 5023 & $9.33 \mathrm{a}$ & $5.44 \mathrm{a}$ \\
$\quad 1995$ & 3026 & $11.76 \mathrm{~b}$ & $10.95 \mathrm{~b}$ & $1.60 \mathrm{a}$ & 2468 & $9.66 \mathrm{~b}$ & $6.40 \mathrm{~b}$ \\
Rango de & & $0.06-$ & $0.06-$ & $0.005-$ & & $0.025-$ & $0.020-$ \\
Error Estandar & & 0.08 & 0.07 & 0.006 & & 0.032 & 0.025 \\
Bimestre: & & & & & & & \\
Ene-Feb & 1571 & $11.31 \mathrm{~b}$ & $10.45 \mathrm{bc}$ & $1.60 \mathrm{~b}$ & 1413 & $9.58 \mathrm{c}$ & $6.16 \mathrm{c}$ \\
Mar-Abr & 1849 & $11.24 \mathrm{~b}$ & $10.37 \mathrm{~b}$ & $1.62 \mathrm{~b}$ & 1640 & $9.70 \mathrm{~d}$ & $5.84 \mathrm{~b}$ \\
May-Jun & 1765 & $10.99 \mathrm{a}$ & $10.16 \mathrm{a}$ & $1.61 \mathrm{~b}$ & 1310 & $9.23 \mathrm{a}$ & $5.48 \mathrm{a}$ \\
Jul-Ago & 965 & $11.85 \mathrm{c}$ & $10.99 \mathrm{~d}$ & $1.55 \mathrm{a}$ & 892 & $9.38 \mathrm{~b}$ & $5.57 \mathrm{a}$ \\
Sep-Oct & 1107 & $11.33 \mathrm{~b}$ & $10.64 \mathrm{c}$ & $1.60 \mathrm{~b}$ & 1035 & $9.45 \mathrm{~b}$ & $5.84 \mathrm{~b}$ \\
Nov-Dic & 1313 & $11.43 \mathrm{~b}$ & $10.69 \mathrm{c}$ & $1.62 \mathrm{~b}$ & 1201 & $9.67 \mathrm{~cd}$ & $6.64 \mathrm{~d}$ \\
Rango de & & $0.07-$ & $0.07-$ & $0.006-$ & & $0.03-$ & $0.024-$ \\
Error Estandar & & 0.11 & 0.10 & 0.009 & & 0.04 & 0.034 \\
\hline
\end{tabular}

TCN= tamaño de la camada al nacer, NLV= número de lechones nacidos vivos, PLN= promedio de peso de los lechones al nacer, NLD= número de lechones destetados, y PLD peso promedio de los lechones la destete. a,b,c, medias de columnas por factor con literales distintas son diferentes entre sí ( $\mathrm{p}<0.05)$.

\section{Revista Biomédica}


Factores asociados a la producción de cerdos.

mínimos y rango de errores estándar para el año y bimestre de parto se presentan en el cuadro 2. En general los indicadores productivos para el año de 1995 fueron mejores que para 1994. El efecto de bimestre fue significativo sobre todas las variables de respuesta estudiadas.

Las marranas de primer y segundo parto tuvieron camadas con pesos menores que aquellas con 3 ó 4 partos (cuadro 3). Sin embargo, al destete no se encontró diferencia en las medias de los tamaños de camada y con respecto a los PLD las marranas de primer y cuarto parto tuvieron los promedios más bajos.

\section{DISCUSIÓN.}

El año de parto tuvo efecto $(\mathrm{p}<0.01)$ sobre todas las variables de respuesta, excepto el PLN. Las causas de estas diferencias, son difíciles de explicar aunque pudieran ser atribuibles a mejoras en las condiciones de manejo con el transcurso del tiempo, resultado de una mayor experiencia en el cuidado de las marranas. Así mismo, las diferencias entre bimestres de parto son parcialmente atribuibles a las condiciones climáticas, aunque los aspectos de manejo de los animales no pueden ser descartados. Efectos del año de servicio en el TCN han sido notificados por $(9,10,14)$ y de la época de servicio por (14).

Las marranas de primer parto tuvieron tamaños de camada al nacer más grandes que las marranas de dos partos, pero menores que las marranas de tres y cuatro partos (cuadro 3). Esto es contrario a lo encontrado en la literatura, que menciona que las marranas primerizas normalmente tienen menores TCN y NLV que las marranas con dos o más partos (10,15-17). Esta diferencia puede explicarse ya que el TC no sólo es una función de la edad sino también del peso y condición corporal de la marrana al parto. Marranas primerizas en buen estad, podrían producir camadas tan grandes al nacer como las marranas adultas.

Al destete, el tamaño de camada de las marranas primerizas fue similar al de las otras marranas pero éstas tuvieron PLD menores que las marranas con dos y tres partos (cuadro 3). Esto puede explicarse ya que en las marranas primerizas existe una menor madurez de la glándula mamaria y por lo tanto una menor capacidad de producción láctea. El hecho de que las marranas con cuatro partos tuvieran PLD menores que las de segundo y tercer parto puede deberse a que los lechones de las primeras se destetaban una edad más temprana (18 días) en comparación a las de segundo y tercer parto (24 días).

En conclusión, los promedios de pesos al

Cuadro 3

Medias de cuadrados mínimos para tamaño de camada y peso de los lechones al nacer y al destete, según el número de parto de la marrana.

\begin{tabular}{|c|c|c|c|c|c|c|c|}
\hline & $\mathrm{N}$ & $\mathrm{TCN}$ & NLV & PLN & $\mathrm{N}$ & NLD & PLD \\
\hline \multicolumn{8}{|c|}{ Número de parto: } \\
\hline 1 & 3257 & $11.09 \mathrm{~b}$ & $10.30 \mathrm{a}$ & $1.48 \mathrm{a}$ & 2863 & $9.47 \mathrm{a}$ & $5.87 \mathrm{~b}$ \\
\hline 2 & 2745 & $10.74 \mathrm{a}$ & $10.15 \mathrm{a}$ & $1.64 b$ & 2497 & $9.48 \mathrm{a}$ & $6.05 \mathrm{c}$ \\
\hline 3 & 1863 & $11.58 \mathrm{c}$ & $10.78 b$ & $1.63 b$ & 1627 & $9.51 \mathrm{a}$ & $6.04 \mathrm{c}$ \\
\hline 4 & 705 & $12.03 \mathrm{~d}$ & $10.97 \mathrm{~b}$ & $1.64 \mathrm{~b}$ & 504 & $9.54 \mathrm{a}$ & $5.74 \mathrm{a}$ \\
\hline \multirow{2}{*}{\multicolumn{2}{|c|}{$\begin{array}{l}\text { Rango de } \\
\text { Error Estándar }\end{array}$}} & $0.06-$ & $0.06-$ & $0.005-$ & & $0.02-$ & 0.020 \\
\hline & & 0.12 & 0.12 & 0.010 & & 0.05 & 0.043 \\
\hline
\end{tabular}

$\mathrm{TCN}=$ tamaño de la camada al nacer, $\mathrm{NLV}=$ número de lechones nacidos vivos, $\mathrm{PLN}=$ promedio de peso de los lechones al nacer, NLD= número de lechones destetados, y PLD peso promedio de los lechones la destete. $a, b, c$, medias de columnas por factor con literales distintas son diferentes entre sí $(p<0.05)$. 


\section{Gómez Medina, JC Segura-Correa, JC Rodríguez-Buenfil.}

nacimiento y al destete de los lechones estuvieron influenciados por el número de parto de las marranas. Así mismo, el año y bimestre de nacimiento fueron importantes fuentes de variación para todas las variables de respuesta estudiadas.

\section{REFERENCIAS.}

1.- French LR, Rutledge JJ, First NL. Effect of age and party on litter size in pigs. J Reprod Fertil 1979; 57:59-60.

2.- Lynch PB, Kearney PA. O'Grandy JF. What influence birth weight of piglets? Farm and Food Res 1982; 13:8899.

3.- Pour M, Povorka F. The effect of parity on the reproductive performance of Czechoslovakian improved White sows used in purebreeding and crossbreeding. Pig News and Information 1983; 4:1.

4.- Lambarri RJ. Parámetros reproductivos de una granja porcina en el Trópico. Tesis de Licenciatura. Facultad de Medicina Veterinaria y Zootecnia. Universidad Autónoma de México. México. 1981.

5.- Peralta RC. Evaluación de la productividad de una granja porcina en el estado de Puebla. Tesis de Licenciatura. Facultad de Medicina Veterinaria y Zootecnia. Universidad Autónoma de México. México. 1981.

6.- Arce HE. Evaluación de una explotación porcina ubicada en Tepejí del Río, Hidalgo. Tesis de Licenciatura. Facultad de Medicina Veterinaria y Zootecnia. Universidad Autónoma de México. México. 1983.

7.- Iñiguez IS. Evaluación de una granja porcina en el estado de San Luis Potosí. Tesis de Licenciatura. Facultad de Medicina Veterinaria y Zootecnia. Universidad Autónoma de México. México. 1983.

8.- Bachtold SJM. Evaluación de la productividad de una granja porcina en el estado de Michoacán. Tesis de Licenciatura. Facultad de Medicina Veterinaria y Zootecnia. Universidad Autónoma de México. México. 1984.

9.- Segura CJC, Segura CVM. Factores genéticos y ambientales que afectan las características predestete en cerdos criados bajo condiciones de trópico húmedo. Rev Mex Prod Anim 1985; 17:13-22.

10.-Segura CJC, Segura CVM. Influencia de algunos factores genéticos y ambientales sobre la eficiencia reproductiva

\section{Revista Biomédica}

de cerdos en una granja de la Chontalpa, Tabasco. Vet Mex 1991; 22:73-6.

11.-English RP, Smith WJ, MacLean A. La cerda: como mejorar su productividad. México: El Manual Moderno; 1981. p. $248-80$.

12.-Rico C, Gómez J. Factores que afectan el comportamiento reproductivo en cerdos. World Rev Anim Prod 1981; 17:49-64.

13.-SAS Institute Inc. SAS/STat User's Guide, Version 6, Fourth edition, Cary North Carolina: SAS Institute Inc.; 1989. vol: 2. p. 891-996.

14.-Leigh AO. Litter performance characteristics of pigs in tropical South-Western Nigeria. 1. Breed differences and effects of some non-genetic sources of variation. Anim Prod 1977; 24:323-31.

15.-Egbunike GN, Steinbach J. Seasonal changes in sow fertility in a humid tropical environment. Anim Breed Abstr 1982; 50:328.

16.-Pond WG, Maner JH. Swine Production in Temperate and Tropical Environments. San Francisco: WH Freeman; 1974. p. 129-52.

17.- Ramirez R, Segura JC. Factores que afectan el comportamiento reproductivo de los cerdos en el noreste de México. 1. Tamaño de la camada y promedio de peso de los lechones. Tec Pec Méx 1991; 30:53-8. 